\title{
On inner dilatations of the mappings with unbounded characteristic
}

\author{
Evgenii A. Sevost'yanov and Ruslan R. Salimov
}

Presented by V. Ya. Gutlyanskiu

\begin{abstract}
For the mappings $f: D \rightarrow D^{\prime}, D, D^{\prime} \subset \mathbb{R}^{n}, n \geq 2$, satisfying certain geometric conditions in the fixed domain $D$, we have proved estimates of the form $K_{I}(x, f) \leq Q(x)$ almost everywhere, where $K_{I}(x, f)$ is the inner dilatation of $f$ at a point $x$, and $Q(x)$ is a fixed real-valued function responsible for the "control" over a distortion of the families of curves in $D$ at a mapping $f$.
\end{abstract}

Keywords. Mappings with bounded and finite distortion, moduli, capacities.

\section{Introduction}

In what follows, $D$ is a domain in $\mathbb{R}^{n}, n \geq 2, m$ is the Lebesgue measure of $\mathbb{R}^{n}$, and the notation $f: D \rightarrow \mathbb{R}^{n}$ assumes that the mapping $f$ given in a domain $D$ is continuous. Hereafter, by a curve $\gamma$, we call a continuous mapping of the segment $[a, b]$ (or an open interval $(a, b)$ ) in $\mathbb{R}^{n}, \gamma:[a, b] \rightarrow \mathbb{R}^{n}$. The family of curves $\Gamma$ means some fixed collection of curves $\gamma$, and $f(\Gamma)=$ $\{f \circ \gamma \mid \gamma \in \Gamma\}$. We recall that a homeomorphism $f: D \rightarrow \overline{\mathbb{R}^{n}}$ in the domain $D \subset \mathbb{R}^{n}, n \geq 2$, $\overline{\mathbb{R}^{n}}=\mathbb{R}^{n} \cup\{\infty\}$, is called a quasiconformal mapping, if

$$
(1 / K) M(\Gamma) \leq M(f(\Gamma)) \leq K M(\Gamma)
$$

for any family $\Gamma$ of curves $\gamma$ in a domain $D$, where $M$ is the conformal modulus of the family of curves (the outer measure defined on families of curves in $\mathbb{R}^{n}$ ), and $K \geq 1$ is some constant (see Definition 13.1 in [16, Sect. 13, Chapt. II]). In other words, the modulus of any family of curves is distorted by at most $K$ times. In this case, for the mapping $f$ to be quasiconformal, it is sufficient that only one inequality on the right-hand side of relation (1.1) be satisfied. Namely, the homeomorphism $f$ is a quasiconformal mapping, as soon as

$$
M(f(\Gamma)) \leq K M(\Gamma)
$$

for any family $\Gamma$ of curves $\gamma$ in a domain $D$ (see Theorem 34.3 in [16, Chapt. IV]). As known, the homeomorphisms satisfying relation (1.2) in $D$ have an almost everywhere nondegenerate Jacobian $J(x, f)$ (see, e.g., Theorem 34.4 in [16]). We define the inner dilatation $K_{I}(x, f)$ of a mapping $f$ at a point $x$ by the ratio

$$
K_{I}(x, f)=\frac{|J(x, f)|}{l\left(f^{\prime}(x)\right)^{n}}
$$

if the Jacobian $J(x, f):=\operatorname{det} f^{\prime}(x) \neq 0 ; K_{I}(x, f)=1$ if $f^{\prime}(x)=0$, and $K_{I}(x, f)=\infty$. The following proposition is valid (see Theorem 34.6 in [16, Chapt. IV]).

Translated from Ukrains'kiǔ Matematychnyı̌ Visnyk, Vol. 8, No. 1, pp. 129-143, January-February, 2011.

Original article submitted June 15, 2010 
Statement 1.1. Assume that the homeomorphism $f: D \rightarrow \overline{\mathbb{R}^{n}}, n \geq 2$, satisfies relation (1.2) in a domain $D$ for any family of curves $\Gamma$. Then, for almost all $x \in D$, the estimate

$$
|J(x, f)| \leq K \cdot l\left(f^{\prime}(x)\right)
$$

where $J(x, f)$ means the Jacobian of a mapping $f$ at a point $x$, and $l\left(f^{\prime}(x)\right):=\min _{h \in \mathbb{R}^{n} \backslash\{0\}} \frac{\left|f^{\prime}(x) h\right|}{|h|}$, is true.

With the regard for the above remark that $J(x, f) \neq 0$ almost everywhere, relation (1.3) can be written in the equivalent form $K_{I}(x, f) \leq K$ for almost all $x \in D$.

Let us now replace relation (1.1) in the definition of the class of mappings under study by an inequality of the form

$$
M(f(\Gamma)) \leq \int_{D} Q(x) \cdot \rho^{n}(x) d m(x),
$$

where $\rho$ is an arbitrary nonnegative Borel function such that any curve $\gamma$ of the family $\Gamma$ has length of at least 1 in the metric $\rho$. In other words, a curvilinear first-kind integral along the curve $\gamma$ satisfies the condition

$$
\int_{\gamma} \rho(x)|d x| \geq 1
$$

for all curves $\gamma \in \Gamma$, and $Q: D \rightarrow[1, \infty]$ is a given function measurable by Lebesgue (see, e.g., [8, Sect. 4.1, Chapt. IV]). The study of inequalities of type (1.4) goes back to L. V. Ahlfors (see, e.g., Theorem 3 in [1, Sect. D, Chapt. I] and to O. Lehto and K. Virtanen (see inequality (6.6) in [7, Sect. 6.3, Chapt. V]). Inequality (1.4) was mentioned by Yu. F. Strugov in [14] in the context of the study of mappings that are quasiconformal in the mean. In the spatial case, the inequality of type (1.4) was established by V. Ya. Gutlyanskii jointly with C. J. Bishop, O. Martio, and M. Vuorinen in work [2] for quasiconformal mappings with the use of detailed strict arguments; in this case, $Q$ is equal to the inner dilatation $K_{I}(x, f)$. In this context, V. M. Miklyukov studied some similar classes, but on the basis of capacities (see [9]). In the case where $Q(x) \leq K$ almost everywhere, relation (1.4) yields inequality (1.2). In the general case where $Q(x)$ can be unbounded, inequality (1.4) means that a distortion of the modulus of the initial family $\Gamma$ occurs, as is said, "with some weight $Q(x)$ ", $M(f(\Gamma)) \leq M_{Q}(\Gamma)$ (see, e.g., works by A. C. Cazacu [3] and M. Cristea [4]). We note that it is quite sufficient sometimes to restrict oneself to some specific families $\Gamma$ in order to determine properties of the mapping $f$ satisfying relations of the form (1.4), rather than to consider all families $\Gamma$ of curves $\gamma$ without exclusion. The main results of the present work are the following two propositions.

Statement 1.2. Let $f: D \rightarrow \overline{\mathbb{R}^{n}}$ be an open discrete mapping satisfying an estimate of the form (1.4) in a domain $D$ for any family $\Gamma$ of curves $\gamma$ in $D$ and any $\rho \in \operatorname{adm} \Gamma$. We assume that $Q \in L_{\text {loc }}^{1}(D)$. Then, for almost all $x \in D$, the inequality

$$
K_{I}(x, f) \leq Q(x)
$$

is valid.

Let $E$ and $F \subset \overline{\mathbb{R}^{n}}$ be any sets. By $\Gamma(E, F, D)$, we denote the family of all curves $\gamma:[a, b] \rightarrow \overline{\mathbb{R}^{n}}$ which join $E$ and $F$ in $D$, i.e., $\gamma(a) \in E, \gamma(b) \in F$ and $\gamma(t) \in D$ at $t \in(a, b)$. In [5, Sect. 13], F. W. Gehring defined a $K$-quasiconformal mapping as a homeomorphism varying the modulus of a 
ring domain by at most $K$ times. In view of the above definition, we consider the following notion. They say that the mapping $f: D \rightarrow \overline{\mathbb{R}^{n}}$ is a ring $Q$-mapping at a point $x_{0} \in D$, if the relation

$$
M\left(f\left(\Gamma\left(S_{1}, S_{2}, A\right)\right)\right) \leq \int_{A} Q(x) \cdot \eta^{n}\left(\left|x-x_{0}\right|\right) d m(x)
$$

is satisfied for any $\operatorname{ring} A=A\left(r_{1}, r_{2}, x_{0}\right)=\left\{x \in \mathbb{R}^{n}: r_{1}<\left|x-x_{0}\right|<r_{2}\right\}, 0<r_{1}<r_{2}<r_{0}$, and for any measurable function $\eta:\left(r_{1}, r_{2}\right) \rightarrow[0, \infty]$ such that

$$
\int_{r_{1}}^{r_{2}} \eta(r) d r \geq 1
$$

We note that condition (1.7) plays the same role as the function $\rho$ in inequality (1.5). Namely, (1.7) is simply a condition of admissibility for a special family of curves $\Gamma\left(S_{1}, S_{2}, A\right)$. They say that the mapping $f: D \rightarrow \overline{\mathbb{R}^{n}}$ is a ring $Q$ mapping in $D$, if relations (1.6)-(1.7) are satisfied at every point $x_{0} \in D$.

Statement 1.3. Let $f: D \rightarrow \overline{\mathbb{R}^{n}}$ be an open discrete mapping that satisfies relations of the form (1.6)-(1.7) at every point $x_{0}$ of a domain $D$. We assume that $Q \in L_{l o c}^{1}(D)$ and $J(x, f) \neq 0$ almost everywhere in $D$. Then, for almost all $x \in D$, the relation

$$
K_{I}(x, f) \leq c_{n} \cdot Q(x),
$$

where the constant $c_{n}$ depends only on $n$, is satisfied.

\section{Preliminary information}

Everywhere below, $B\left(x_{0}, r\right)=\left\{x \in \mathbb{R}^{n}:\left|x-x_{0}\right|<r\right\}$. The mapping $f: D \rightarrow \overline{\mathbb{R}^{n}}$ is called discrete, if the preimage $f^{-1}(y)$ of every point $y \in \mathbb{R}^{n}$ consists of isolated points, and it is open, if the image of any open set $U \subset D$ is an open set in $\mathbb{R}^{n}$. The following definitions can be found e.g., in [16, Sect. 1-6, Chapt. I]. A Borel function $\rho: \mathbb{R}^{n} \rightarrow[0, \infty]$ is called admissible for the family $\Gamma$ of curves $\gamma$ in $\mathbb{R}^{n}$, if relation (1.5) is satisfied for all curves $\gamma \in \Gamma$. In this case, we write $\rho \in \operatorname{adm} \Gamma$. By the modulus of a family of curves $\Gamma$, we call the quantity

$$
M(\Gamma)=\inf _{\rho \in \operatorname{adm} \Gamma} \int_{D} \rho^{n}(x) d m(x) .
$$

To a some extent, the properties of a modulus are analogous to those of the Lebesgue measure $m$ in $\mathbb{R}^{n}$. Namely, the modulus of an empty family of curves is equal to zero, $M(\varnothing)=0$, has the property of monotonicity relative to the families of curves $\Gamma_{1}$ and $\Gamma_{2}, \Gamma_{1} \subset \Gamma_{2} \Rightarrow M\left(\Gamma_{1}\right) \leq M\left(\Gamma_{2}\right)$, and has the property of semiadditivity:

$$
M\left(\bigcup_{i=1}^{\infty} \Gamma_{i}\right) \leq \sum_{i=1}^{\infty} M\left(\Gamma_{i}\right)
$$

(see Theorem 6.2 in [16]). They say that the family of curves $\Gamma_{1}$ is minorized by a family $\Gamma_{2}$, i.e., $\Gamma_{1}>\Gamma_{2}$, if, for any curve $\gamma \in \Gamma_{1}$, there exists a subcurve that belongs to the family $\Gamma_{2}$. It is well known that

$$
\Gamma_{1}>\Gamma_{2} \Rightarrow M\left(\Gamma_{1}\right) \leq M\left(\Gamma_{2}\right)
$$

(see Theorem 6.4 in [16]). 
The following important definitions can be found in [11, Sect. 3, Chapt. II]. Let $f: D \rightarrow \mathbb{R}^{n}, \beta$ : $[a, b) \rightarrow \mathbb{R}^{n}$ be some curve, and let $x \in f^{-1}(\beta(a))$. The curve $\alpha:[a, c) \rightarrow D$ is called a maximal lifting of the curve $\beta$ at the mapping $f$ started at a point $x$, if

(i) $\alpha(a)=x$;

(ii) $f \circ \alpha=\left.\beta\right|_{[a, c)}$;

(iii) if $c<c^{\prime} \leq b$, then there exists no curve $\alpha^{\prime}:\left[a, c^{\prime}\right) \rightarrow D$ such that $\alpha=\left.\alpha^{\prime}\right|_{[a, c)}$ and $f \circ \alpha^{\prime}=\left.\beta\right|_{\left[a, c^{\prime}\right)}$.

Let $f$ be an open discrete mapping, and let $x \in f^{-1}(\beta(a))$. Then the curve $\beta:[a, b) \rightarrow \mathbb{R}^{n}$ has a maximal lifting at the mapping $f$ started at a point $x$ (see Corollary 3.3 in [11, Chapt. II]). The pair $E=(A, C)$, where $A$ is an open set in $\mathbb{R}^{n}$ and $C$ is a compact subset $A$, is called a condenser in $\mathbb{R}^{n}, n \geq 2$. The mapping $f: D \rightarrow \mathbb{R}^{n}$ is called absolutely continuous on lines, i.e., $f \in A C L$, if all coordinate functions $f=\left(f_{1}, \ldots, f_{n}\right)$ are absolutely continuous on almost all lines parallel to the coordinate axes in any $n$-dimensional parallelepiped $P$ such that $\bar{P} \subset D$ and its edges are parallel to the coordinate axes. It is known that if $f \in A C L$, then $f$ has almost everywhere partial derivatives in $D$. As the capacity of a condenser $E$, we call the quantity

$$
\operatorname{cap} E=\operatorname{cap}(A, C)=\inf _{u \in W_{0}(E)} \int_{A}|\nabla u|^{n} d m(x),
$$

where $W_{0}(E)=W_{0}(A, C)$ is the family of nonnegative continuous functions $u: A \rightarrow \mathbb{R}$ with compact support in $A$ and such that $u(x) \geq 1$ at $x \in C$ and $u \in A C L$. It is known that, for an arbitrary condenser $E=(A, C)$, the following relation is true:

$$
\operatorname{cap} E \geq \frac{\left(\inf m_{n-1} S\right)^{n}}{[m(A \backslash C)]^{n-1}} .
$$

Here, $m_{n-1} S$ is the $(n-1)$-dimensional Lebesgue measure of a $C^{\infty}$-manifold $S$ which is the boundary $S=\partial U$ of a bounded open set $U$ that contains $C$ and is contained together with its closure $\bar{U}$ in $A$. In (2.2), the infimum was taken over such all $S$ (see Proposition 5 in [6]).

Let $E=(A, C)$ be any condenser in $\mathbb{R}^{n}$. Then, by $\Gamma_{E}$, we denote the family of all curves of the type $\gamma:[a, b) \rightarrow A$ with $\gamma(a) \in C$ such that $|\gamma| \cap(A \backslash F) \neq \varnothing$ for any compact set $F \subset A$. In other words, the family $\Gamma_{E}$ for the condenser $E=(A, C)$ consists of those and only those curves that start in $C$, lie in $A$, and, at the same time, belong completely to none of the fixed compact sets inside $A$. For a bounded set $A$, such curves must "approach" the boundary of $A$. However, they should not be rectifiable and, generally speaking, should not tend to something.

Proposition 2.1 (see [11, Proposition 10.2, Chapt. II]). The equality

$$
\operatorname{cap} E=M\left(\Gamma_{E}\right)
$$

holds.

For a mapping $f: D \rightarrow \mathbb{R}^{n}$ having partial derivatives in $D$ almost everywhere,

$$
\left\|f^{\prime}(x)\right\|=\max _{h \in \mathbb{R}^{n} \backslash\{0\}} \frac{\left|f^{\prime}(x) h\right|}{|h|} .
$$


The outer dilatation of the mapping $f$ at a point $x$ is the quantity

$$
K_{O}(x, f)=\frac{\left\|f^{\prime}(x)\right\|^{n}}{|J(x, f)|}
$$

if $J(x, f) \neq 0, K_{O}(x, f)=1$, if $f^{\prime}(x)=0$, and $K_{O}(x, f)=\infty$ at the rest points. The linear dilatation $f$ at a point $x$ is the quantity

$$
H(x, f)=\sqrt[n]{K_{I}(x, f) K_{O}(x, f)} .
$$

We assume that the mapping $f: D \rightarrow \mathbb{R}^{n}$ is differentiable at the point $x_{0} \in D$, and the Jacobi matrix $f^{\prime}\left(x_{0}\right)$ is nonsingular, $J(x, f)=\operatorname{det} f^{\prime}\left(x_{0}\right) \neq 0$. Then there exist the systems of vectors $e_{1}, \ldots, e_{n}$ and $\widetilde{e_{1}}, \ldots, \widetilde{e_{n}}$ and the positive numbers $\lambda_{1}\left(x_{0}\right), \ldots, \lambda_{n}\left(x_{0}\right), \lambda_{1}\left(x_{0}\right) \leq \cdots \leq \lambda_{n}\left(x_{0}\right)$, such that $f^{\prime}\left(x_{0}\right) e_{i}=$ $\lambda_{i}\left(x_{0}\right) \widetilde{e_{i}}$ (see Theorem 2.1 in [10, Chapt. I]). In this case, $\lambda_{1}^{2}\left(x_{0}\right), \ldots, \lambda_{n}^{2}\left(x_{0}\right)$ are the eigenvalues of the symmetric mapping $\left(f^{\prime}\left(x_{0}\right)\right)^{*} f^{\prime}\left(x_{0}\right)$ (see Theorem 2.2 in [10, Chapt. I]),

$$
\begin{gathered}
\left|J\left(x_{0}, f\right)\right|=\lambda_{1}\left(x_{0}\right) \ldots \lambda_{n}\left(x_{0}\right), \quad\left\|f^{\prime}\left(x_{0}\right)\right\|=\lambda_{n}\left(x_{0}\right), \\
l\left(f^{\prime}\left(x_{0}\right)\right)=\lambda_{1}\left(x_{0}\right), \\
K_{O}\left(x_{0}, f\right)=\frac{\lambda_{n}^{n}\left(x_{0}\right)}{\lambda_{1}\left(x_{0}\right) \cdots \lambda_{n}\left(x_{0}\right)}, \quad K_{I}\left(x_{0}, f\right)=\frac{\lambda_{1}\left(x_{0}\right) \cdots \lambda_{n}\left(x_{0}\right)}{\lambda_{1}^{n}\left(x_{0}\right)}
\end{gathered}
$$

(see relation (2.5) and additional comments on p. 21 in [10, Sect. 2.1, Chapt. I]). In addition, the above-presented formulas yield

$$
K_{I}(x, f) \leq K_{O}^{n-1}(x, f), \quad K_{O}(x, f) \leq K_{I}^{n-1}(x, f)
$$

(see relations (2.7) and (2.8) in [10, Sect. 2.1, Chapt. I]), and $K_{I}(x, f) \geq 1$ and $K_{O}(x, f) \geq 1$ in all domains, where these quantities are well-defined.

The above-mentioned numbers $\lambda_{1}\left(x_{0}\right), \ldots \lambda_{n}\left(x_{0}\right)$ are called principal values, and the vectors $e_{1}, \ldots$, $e_{n}$ and $\widetilde{e_{1}}, \ldots, \widetilde{e_{n}}$ are called principal vectors of the mapping $f^{\prime}\left(x_{0}\right)$ (see the relevant comment in $[10$, Sect. 2.1, Chapt. I] after the proof of Theorem 2.2). Of course, the principal vectors and the principal values depend on the point $x_{0}$ and on the mapping $f$. However, in order to simplify the representation, we will omit " $\left(x_{0}\right)$ " in what follows, if no misunderstanding occurs.

\section{On the estimate of the inner dilatation of open discrete ring $Q$-mappings}

Theorem 3.1. Let $f: D \rightarrow \overline{\mathbb{R}^{n}}$ be an open discrete mapping that satisfies relations of the form (1.6)-(1.7) at every point $x_{0}$ of the domain $D$. We assume that $Q \in L_{l o c}^{1}(D)$ and $J(x, f) \neq 0$ almost everywhere. Then, at almost all $x \in D$, the relation

$$
K_{I}(x, f) \leq c_{n} \cdot Q(x),
$$

where the constant $c_{n}$ depends only on $n$, is true.

Proof. According to Theorem 3.2 in [13], $f$ is differentiable almost everywhere in $D$. Without any loss of generality, we consider that $\infty \notin D^{\prime}=f(D)$. At every point $x \in D$ where the mapping $f$ is differentiable and $J(x, f) \neq 0$, we consider the condenser $E_{r}=\left(A_{r}, G_{r}\right)$, where $A_{r}=\{y:|x-y|<2 r\}$ and $G_{r}=\{y:|x-y| \leq r\}$. Since $f$ is an open continuous mapping, $f\left(E_{r}\right)$ is also a condenser in $\mathbb{R}^{n}$. Let $\Gamma_{E_{r}}$ and $\Gamma_{f\left(E_{r}\right)}$ be the families of curves in the meaning of designations given prior to Proposition 2.1, 
and let $\Gamma_{r}^{*}$ be the family of maximal lifting of $\Gamma_{f\left(E_{r}\right)}$ at the mapping $f$ started in $G_{r}$. We will show that $\Gamma_{r}^{*} \subset \Gamma_{E_{r}}$.

Assume the contrary. Let there exist a curve $\beta:[a, b) \rightarrow \mathbb{R}^{n}$ of the family $\Gamma_{f\left(E_{r}\right)}$ for which the corresponding maximal lifting $\alpha:[a, c) \rightarrow A_{r}$ belongs to some compact set $K$ inside of $A_{r}$. Hence, its closure $\bar{\alpha}$ is a compact set in $A_{r}$. We note that $c \neq b$, since $\bar{\beta}$ is a compact set in $f\left(A_{r}\right)$ otherwise, which contradicts the condition $\beta \in \Gamma_{f\left(E_{r}\right)}$. Let us consider the limit set of a curve $\alpha(t)$ at $t \rightarrow c-0$ :

$$
G=\left\{x \in \mathbb{R}^{n}: x=\lim _{k \rightarrow \infty} \alpha\left(t_{k}\right)\right\}, \quad t_{k} \in[a, c), \quad \lim _{k \rightarrow \infty} t_{k}=c,
$$

where $t_{k} \rightarrow c-0$ monotonically. For $x \in G$ by virtue of the continuity of $f$, we have $f\left(\alpha\left(t_{k}\right)\right) \rightarrow f(x)$ as $k \rightarrow \infty$, where $t_{k} \in[a, c), t_{k} \rightarrow c$ as $k \rightarrow \infty$. However, $f\left(\alpha\left(t_{k}\right)\right)=\beta\left(t_{k}\right) \rightarrow \beta(c)$ as $k \rightarrow \infty$. This allows us to conclude that $f$ is constant on $G$ in $A_{r}$. On the other hand, in the compact set $\bar{\alpha}$ (see [17, Chapt. I]),

$$
G=\bigcap_{k=1}^{\infty} \overline{\alpha\left(\left[t_{k}, c\right)\right)}=\limsup _{k \rightarrow \infty} \alpha\left(\left[t_{k}, c\right)\right)=\liminf _{k \rightarrow \infty} \alpha\left(\left[t_{k}, c\right)\right) \neq \varnothing
$$

by the Cantor condition in view of the monotonicity relative a sequence of connected sets $\alpha\left(\left[t_{k}, c\right)\right)$. Hence, $G$ is connected (see [17, Sect. 9.12, Chapt. I]). Thus, by virtue of the discreteness of $f$, the set $G$ cannot consist of two and more points, and the curve $\alpha:[a, c) \rightarrow A_{r}$ is continued to the closed curve $\alpha:[a, c] \rightarrow K \subset A_{r}$, and $f(\alpha(c))=\beta(c)$. Again by Corollary 3.3 in [11, Chapt. II], we can construct the maximal lifting $\alpha^{\prime}$ of a curve $\left.\beta\right|_{[c, b)}$ started at the point $\alpha(c)$. Joining the liftings $\alpha$ and $\alpha^{\prime}$, we obtain a new lifting $\alpha^{\prime \prime}$ of the curve $\beta$ that is defined on $\left[a, c^{\prime}\right), c^{\prime} \in(c, b)$, which contradicts the maximality of the lifting $\alpha$. Thus, $\Gamma_{r}^{*} \subset \Gamma_{E_{r}}$. We note that $\Gamma_{f\left(E_{r}\right)}>f\left(\Gamma_{r}^{*}\right)$, and, hence, Proposition 2.1 and the property of minorizing (2.1) yield

$$
\operatorname{cap} f\left(E_{r}\right)=M\left(\Gamma_{f\left(E_{r}\right)}\right) \leq M\left(f\left(\Gamma_{r}^{*}\right)\right) \leq M\left(f\left(\Gamma_{E_{r}}\right)\right) .
$$

We note that the mapping $f$ satisfies, by condition, relations (1.6)-(1.7), i.e., it is a ring $Q$-mapping. Then relation (3.2) yields

$$
\operatorname{cap} f\left(E_{r}\right) \leq \int_{r<|x-y|<2 r} Q(y) \eta^{n}(|x-y|) d m(y)
$$

for any nonnegative measurable function $\eta:(r, 2 r) \rightarrow[0, \infty]$ such that $\int_{r}^{2 r} \eta(t) d t \geq 1$. In particular, let us consider the one-parameter family of real-valued functions

$$
\eta_{r}(t)= \begin{cases}\frac{1}{r}, & \text { if } t \in(r, 2 r) \\ 0, & \text { if } t \in \mathbb{R} \backslash(r, 2 r)\end{cases}
$$

Then

$$
\operatorname{cap} f\left(E_{r}\right) \leq \frac{2^{n} \Omega_{n}}{m\left(A_{r}\right)} \int_{A_{r}} Q(y) d m(y) .
$$

Here $\Omega_{n}$ denotes a volume of the unit ball $\mathbb{B}^{n}$ in $\mathbb{R}^{n}$. On the other hand, with regard for inequality (2.2), we obtain

$$
\operatorname{cap} f\left(E_{r}\right) \geq \frac{\left(\inf m_{n-1} S\right)^{n}}{\left[m\left(f\left(A_{r}\right) \backslash f\left(G_{r}\right)\right)\right]^{n-1}},
$$


where inf is taken over all possible $C^{\infty}$-manifolds $S$ which are the boundary $S=\partial U$ of a bounded open set $U$ that contains $f\left(G_{r}\right)$ and is contained along with its closure $\bar{U}$ in $f\left(A_{r}\right)$. Combining (3.3) and (3.4), we obtain

$$
\left(\inf m_{n-1} S\right)^{n} \leq \frac{2^{n} \Omega_{n}\left[m\left(f\left(A_{r}\right) \backslash f\left(G_{r}\right)\right)\right]^{n-1}}{m\left(A_{r}\right)} \int_{A_{r}} Q(y) d m(y) .
$$

At $r \rightarrow 0$, the set $f\left(G_{r}\right)$ is, to within $o(r)$, an ellipsoid $f^{\prime}\left(G_{r}\right)$ that is the image of the ball $G_{r}$ at the linear mapping $f^{\prime}$. If the given ellipsoid has semiaxes $0<a_{1} r \leq \cdots \leq a_{n} r$, then $m\left(f^{\prime}\left(G_{r}\right)\right)=$ $\Omega_{n} a_{1} \cdots a_{n} r^{n}=\Omega_{n} J(x, f) r^{n}$ (see relations (2.3)). Let us position the ellipsoid so that its center coincides with the coordinate origin, and the principal directions coincide with the coordinate axes $e_{1}, \ldots, e_{n}$. Then the area of its surface admits a lower bound

$$
m_{n-1}\left(\partial f^{\prime}\left(G_{r}\right)\right) \geq 2 m_{n-1}\left(\operatorname{Pr}_{1}\left(f^{\prime}\left(G_{r}\right)\right)\right)=2 \Omega_{n-1} \cdot a_{2} \cdots a_{n} r^{n-1}=2 \Omega_{n-1} \cdot \frac{J(x, f)}{l\left(f^{\prime}(x)\right)} r^{n-1},
$$

where $\operatorname{Pr}_{1}(\cdot)$ means the projection onto a hyperplane perpendicular to the vector $e_{1}$. Hence, in view of $(3.5),(3.6)$, and the relation $J(x, f) \neq 0$, we obtain

$$
\begin{aligned}
{\left[2 \Omega_{n-1} \cdot \frac{J(x, f)}{l\left(f^{\prime}(x)\right)} r^{n-1}-o\left(r^{n-1}\right)\right]^{n} \leq\left[m_{n-1} \partial f^{\prime}\left(G_{r}\right)-o\left(r^{n-1}\right)\right]^{n} } & \\
& \leq \frac{2^{n} \Omega_{n}\left[m\left(f\left(A_{r}\right) \backslash f\left(G_{r}\right)\right)\right]^{n-1}}{m\left(A_{r}\right)} \int_{A_{r}} Q(y) d m(y) .
\end{aligned}
$$

Dividing inequality (3.7) by $r^{n(n-1)}$, turning $r$ to 0 , and applying the Lebesgue theorem of the differentiability of indefinite integrals (see Theorem 5.4 in [12, Chapt. IV]), we obtain

$$
\left[\frac{J(x, f)}{l\left(f^{\prime}(x)\right)}\right]^{n} \leq[J(x, f)]^{n-1} c_{n} \cdot Q(x)
$$

for almost all $x \in D$. By condition, $J(x, f) \neq 0$ almost everywhere. Hence,

$$
K_{I}(x, f)=\frac{J(x, f)}{\left(l\left(f^{\prime}(x)\right)\right)^{n}} \leq c_{n} \cdot Q(x)
$$

for almost all $x \in D$. Theorem 3.1 is proved.

Corollary 3.1. Let $f: D \rightarrow \overline{\mathbb{R}^{n}}$ be an open discrete mapping that satisfies relations of the form (1.6)-(1.7) at every point $x_{0}$ of the domain $D$. We assume that $Q \in L_{l o c}^{1}(D)$ and $J(x, f) \neq 0$ almost everywhere. Then

$$
H(x, f) \leq c_{n} \cdot Q(x)
$$

almost everywhere, where the constant $c_{n}$ depends only on $n$.

Corollary 3.2. Let $f: D \rightarrow \overline{\mathbb{R}^{n}}$ be an open discrete mapping that satisfies relations of the form (1.6)-(1.7) at every point $x_{0}$ of the domain $D$. We assume that $Q \in L_{l o c}^{1}(D)$ and $J(x, f) \neq 0$ almost everywhere. Then $H(x, f) \in L_{l o c}^{1}(D)$ and $K_{I}(x, f) \in L_{l o c}^{1}(D)$. 


\section{On the estimate of the inner dilatation of open discrete $Q$-mappings}

In this section, we will study the mappings satisfying an estimate of the form (1.4) which is stronger than (1.6). Below, we will show that, for the above-indicated mappings, we can take $c_{n} \equiv 1$ in an inequality of the form (3.1) which is also valid for mappings of the form (1.4). Such an estimate will be exact since the inner dilatation is always greater or equal than 1 (see Section 2).

Theorem 4.1. Let $f: D \rightarrow \overline{\mathbb{R}^{n}}$ be an open discrete mapping that satisfies an estimate of the form (1.4) in the domain $D$ for any family $\Gamma$ of curves $\gamma$ in the domain $D$ and arbitrary $\rho \in \operatorname{adm} \Gamma$. We assume that $Q \in L_{l o c}^{1}(D)$. Then, for almost all $x \in D$, the relation

$$
K_{I}(x, f) \leq Q(x)
$$

is satisfied.

Proof. Without any loss of generality, we may consider that $\infty \notin D^{\prime}=f(D)$. According to Theorem 3.2 and Corollary 4.4 in [13], $f$ is differentiable almost everywhere, and $J(x, f) \neq 0$. By $\Phi(A)$, we denote a function of the set $A \subset D$ defined as follows:

$$
\Phi(A)=\int_{A} Q(x) d m(x) .
$$

We note that, by condition, the function $Q \in L_{l o c}^{1}(D)$. Then, by the Lebesgue theorem, the function $\Phi$ is differentiable in the generalized sense at almost every point $x_{0} \in D$ (see, e.g., Theorem 5.4 in $[12$, Chapt. IV]), and the derivative $D \Phi(x)=Q(x)$ for almost all $x \in D$ (there, see Theorem 6.3 and the notion of generalized differentiability of a function of the set at a point). By $E_{1}$, we denote the set of all $x \in D$ where $\Phi$ is differentiable in the generalized sense and $D \Phi(x)=Q(x)$. By $E_{2}$, we denote the set of all $x \in D$ where the mapping itself $f$ is differentiable and nondegenerate. To prove the assertion of the theorem, it is sufficient to show that (4.1) is valid for all $x \in E_{0}=E_{1} \cup E_{2}$.

Let us fix an arbitrary point $x_{0} \in E_{0}$. Without any loss of generality, we may consider that $x_{0}=0$ and $f\left(x_{0}\right)=0$. Let $e_{1}, \ldots, e_{n}, \widetilde{e_{1}}, \ldots, \widetilde{e_{n}}$ and $\lambda_{1}, \ldots, \lambda_{n}$ be, respectively, the principal vectors and principal values of the mapping $f^{\prime}(0), \lambda_{n} \geq \lambda_{n-1} \geq \cdots \geq \lambda_{1}>0$. By the rotation transformation of the image and the preimage, we can obtain $e_{i}=(0, \ldots, 0, \underbrace{1}_{i}, 0, \ldots, 0)=\widetilde{e}_{i}$. We should verify that

$$
\frac{\lambda_{2} \cdots \lambda_{n}}{\lambda_{1}^{n-1}} \leq Q(0)
$$

since $|J(0, f)|=\lambda_{1} \cdots \lambda_{n}$ and $l\left(f^{\prime}(0)\right)=\lambda_{1}$. Let us fix the parameter $t>0$ arbitrarily, and let us choose a number $r>0$ so that the condenser $E:=(A, C)$, where $C=\left\{x: x_{1}=0,\left|x_{i}\right| \leq r, i=\right.$ $2, \ldots, n\}$ and $A=\left\{x:\left|x_{1}\right|<r t \lambda_{1},\left|x_{i}\right|<r+r t \lambda_{i}, i=2, \ldots, n\right\}$ lies in the domain $D$. We note that

$$
m(A)=2^{n} \lambda_{1} r t \prod_{i=2}^{n}\left(r+r t \lambda_{i}\right)
$$

and

$$
\operatorname{dist}(C, \partial A)=r t \lambda_{1}
$$

Since $E=(A, C)$ is a condenser in $D$, we have that $f(E)=(f(A), f(C))$ is a condenser in $D^{\prime}=f(D)$ due to the openness and continuity of $f$. Let $\Gamma_{E}$ and $\Gamma_{f(E)}$ be the families of curves in the meaning of 
Proposition 2.1, and let $\Gamma^{*}$ be the family of maximal liftings $\Gamma_{f(E)}$ at a mapping $f$ started in $C$. As in the proof of Theorem 3.1, we have $\Gamma^{*} \subset \Gamma_{E}$. We note that $\Gamma_{f(E)}>f\left(\Gamma^{*}\right)$, and, hence, the mapping $f$ satisfies relation (1.4) by the condition of the theorem. Then the relation

$$
\operatorname{cap} f(E)=M\left(\Gamma_{f(E)}\right) \leq M\left(f\left(\Gamma^{*}\right)\right) \leq M\left(f\left(\Gamma_{E}\right)\right)
$$

yields

$$
\operatorname{cap}(f(A), f(C)) \leq \int_{D} Q(x) \cdot \rho^{n}(x) d m(x)
$$

for any admissible function $\rho \in \operatorname{adm} \Gamma_{E}$. We note that the function

$$
\rho(x)= \begin{cases}\frac{1}{\operatorname{dist}(C, \partial A)}, & x \in A \backslash C, \\ 0, & x \notin A \backslash C\end{cases}
$$

is admissible for the family $\Gamma_{E}$. Thus, by virtue of (4.4),

$$
\operatorname{cap}(f(A), f(C)) \leq \frac{1}{(\operatorname{dist}(C, \partial A))^{n}} \int_{A} Q(x) d m(x) .
$$

On the other hand, in view of inequality (2.2), relation (4.5) yields

$$
\frac{\left(\inf m_{n-1} S\right)^{n}}{[m(f(A))]^{n-1}} \leq \frac{1}{(\operatorname{dist}(C, \partial A))^{n}} \int_{A} Q(x) d m(x),
$$

where $m_{n-1} S$ means the $(n-1)$-dimensional area of a $C^{\infty}$-manifold $S$ which is the boundary of an open set $U$ that contains $f(C)$ and is contained together with its closure $\bar{U}$ in $f(A)$. In this case, the infimum in (4.6) is taken over all such $S$. Let us estimate the fraction in inequality (4.6), by basing on the property of the differentiability of the mapping $f$ at zero. Fixing $0<\varepsilon<\lambda_{1}$ arbitrarily, we choose $r>0$ so small that $\left|f(x)-f^{\prime}(0) x\right|<\varepsilon r$ at $x \in A$. Then the set $f(A)$ is contained in the parallelepiped

$$
V=\left\{y:\left|y_{1}\right| \leq r t \lambda_{1}^{2}+\varepsilon r,\left|y_{i}\right| \leq r \lambda_{i}+r t \lambda_{i}^{2}+\varepsilon r, i=2, \ldots, n\right\},
$$

and the projection of the set $f(C)$ onto the subspace $y_{1}=0$ contains an $(n-1)$-dimensional parallelepiped

$$
V_{0}=\left\{y: y_{1}=0,\left|y_{i}\right| \leq r \lambda_{i}-\varepsilon r, i=2, \ldots, n\right\}
$$

Therefore,

$$
m(f(A)) \leq m(V)=2^{n} r^{n}\left(t \lambda_{1}^{2}+\varepsilon\right) \prod_{i=2}^{n}\left(\lambda_{i}+t \lambda_{i}^{2}+\varepsilon\right)
$$

and

$$
m_{n-1} S \geq 2 m_{n-1} V_{0}=2^{n} r^{n-1} \prod_{i=2}^{n}\left(\lambda_{i}-\varepsilon\right) .
$$

Hence, substituting the obtained estimates of the above-indicated quantities in inequality (4.6) and taking (4.2) and (4.3) into account, we obtain

$$
\frac{\left(2^{n} r^{n-1} \prod_{i=2}^{n}\left(\lambda_{i}-\varepsilon\right)\right)^{n}}{\left(2^{n} r^{n}\left(t \lambda_{1}^{2}+\varepsilon\right) \prod_{i=2}^{n}\left(\lambda_{i}+t \lambda_{i}^{2}+\varepsilon\right)\right)^{n-1}} \leq \frac{2^{n} \lambda_{1} r t \prod_{i=2}^{n}\left(r+r t \lambda_{i}\right)}{r^{n} t^{n} \lambda_{1}^{n}} \frac{1}{m(A)} \int_{A} Q(x) d m(x) .
$$


This yields

$$
\frac{\left(\prod_{i=2}^{n}\left(\lambda_{i}-\varepsilon\right)\right)^{n}}{\left(\left(t \lambda_{1}^{2}+\varepsilon\right) \prod_{i=2}^{n}\left(\lambda_{i}+t \lambda_{i}^{2}+\varepsilon\right)\right)^{n-1}} \leq \frac{\prod_{i=2}^{n}\left(1+t \lambda_{i}\right)}{t^{n-1} \lambda_{1}^{n-1}} \frac{1}{m(A)} \int_{A} Q(x) d m(x) .
$$

As $r \rightarrow 0$, we have

$$
\frac{\left(\prod_{i=2}^{n}\left(\lambda_{i}-\varepsilon\right)\right)^{n}}{\left(\left(t \lambda_{1}^{2}+\varepsilon\right) \prod_{i=2}^{n}\left(\lambda_{i}+t \lambda_{i}^{2}+\varepsilon\right)\right)^{n-1}} \leq \frac{\prod_{i=2}^{n}\left(1+t \lambda_{i}\right)}{t^{n-1} \lambda_{1}^{n-1}} Q(0)
$$

As $\varepsilon \rightarrow 0$, we obtain

$$
\frac{\left(\prod_{i=2}^{n} \lambda_{i}\right)^{n}}{\left(t \lambda_{1}^{2} \prod_{i=2}^{n}\left(\lambda_{i}+t \lambda_{i}^{2}\right)\right)^{n-1}} \leq \frac{\prod_{i=2}^{n}\left(1+t \lambda_{i}\right)}{t^{n-1} \lambda_{1}^{n-1}} Q(0) .
$$

Then, by multiplying both sides of the inequality by $t^{n-1}$ and by passing to the limit as $t \rightarrow 0$, we obtain

$$
\frac{\prod_{i=2}^{n} \lambda_{i}}{\lambda_{1}^{n-1}} \leq Q(0) .
$$

Hence, Theorem 4.1 is completely proved.

Corollary 4.1. Let $f: D \rightarrow \overline{\mathbb{R}^{n}}$ be an open discrete mapping that satisfies an estimate of the form (1.4) in the domain $D$ for any family $\Gamma$ of curves $\gamma$ in the domain $D$ and arbitrary $\rho \in \operatorname{adm} \Gamma$. We assume that $Q \in L_{l o c}^{1}(D)$. Then $Q(x) \geq 1$ for almost all $x \in D$.

Corollary 4.2. Let $f: D \rightarrow \overline{\mathbb{R}^{n}}$ be an open discrete mapping that satisfies an estimate of the form (1.4) in the domain $D$ for any family $\Gamma$ of curves $\gamma$ in the domain $D$ and arbitrary $\rho \in \operatorname{adm} \Gamma$. We assume that $Q \in L_{l o c}^{1}(D)$. Then $K_{O}(x, f) \leq Q^{n-1}(x)$ for almost all $x \in D$.

Corollary 4.3. Let $f: D \rightarrow \overline{\mathbb{R}^{n}}$ be an open discrete mapping that satisfies an estimate of the form (1.4) in the domain $D$ for any family of curves $\Gamma$ in the domain $D$ and arbitrary $\rho \in \operatorname{adm} \Gamma$. We assume that $Q \in L_{l o c}^{1}(D)$. Then, for almost all $x \in D$,

$$
H(x, f) \leq Q(x)
$$

\section{Final remarks}

We assume that the inequality $K_{O}(x, f) \leq C_{n} \cdot Q^{n-1}(x)$ with $C_{n}=1$ (see, e.g., [13]) and the inequality $K_{I}(x, f) \leq c_{n} Q(x)$ with $c_{n}=1$ for the mappings that satisfy (1.6)-(1.7) at every point $x_{0} \in D$ are no true, generally speaking. In other words, we assume that the class of ring $Q$-mappings is wider than the class of $Q$-mappings. Another open question consists in the present conditions for "openness" and "discreteness" of the mapping $f$. The studies of the dilatations of mappings that are not open and discrete will require to apply a technique that is different from the modulus-based one.

P.S. The present work is carried out in the fairway of the studies initiated by the famous mathematician G. D. Suvorov who considered that "the ideal (and purpose!) of the theory of functions should be the 
attainment of such a situation where we shall possess a large number of various classes of functions and a developed catalog of properties (metric and topological ones) for each class" (see [15, p. 325]).

\section{REFERENCES}

1. L. V. Ahlfors, Lectures on Quasiconformal Mappings, Van Nostrand-Reinhold, Princeton, 1966.

2. C. J. Bishop, V. Ya. Gutlyanskii, O. Martio, and M. Vuorinen, "On conformal dilatation in space," Int. J. Math. and Math. Sci., 22, 1397-1420 (2003).

3. A. C. Cazacu, "On the length-area dilatation," Compl. Var. Theory Appl., 50, No. 7-11, 765-776 (2005).

4. M. Cristea, "Local homeomorphisms having local $A C L^{n}$ inverses," Compl. Var. Ellipt. Equ., 53, No. 1, 77-99 (2008).

5. F. W. Gehring, "Rings and quasiconformal mappings in space," Trans. Amer. Math. Soc., 103, 353-393 (1962).

6. V. I. Kruglikov, "Capacity of condensers and spatial mappings quasiconformal in the mean," Mat. Sb., 130, No. 2, 185-206 (1986).

7. O. Lehto and K. Virtanen, Quasiconformal Mappings in the Plane, Springer, New York, 1973.

8. O. Martio, V. Ryazanov, U. Srebro, and E. Yakubov, Moduli in Modern Mapping Theory, Springer Science + Business Media, New York, 2009.

9. V. M. Miklyukov, The Conformal Mapping of an Irregular Surface and Its Applications [in Russian], Volgograd State Univ., Volgograd, 2005.

10. Yu. G. Reshetnyak, Spatial Mappings with Bounded Distortion [in Russian], Nauka, Novosibirsk, 1982.

11. S. Rickman, Quasiregular Mappings, Springer, Berlin, 1993.

12. S. Saks, Theory of the Integral, New York, Dover, 1964.

13. R. Salimov and E. Sevost'yanov, "ACL and differentiability of the open discrete ring mappings," Compl. Var. Ellipt. Equ., 55, No. 1-3, 49-59 (2010).

14. Yu. F. Strugov, "Compactness of the classes of mappings quasiconformal in the mean," DAN SSSR, 243, No. 4, 859-861 (1978).

15. G. D. Suvorov, On the Art of Mathematical Studies [in Russian], TEAN, Donetsk, 1999.

16. J. Väisälä, Lectures on n-Dimensional Quasiconformal Mappings, Springer, Berlin, 1971.

17. G. T. Whyburn, Analytic Topology, Amer. Math. Soc., New York, 1942.

Evgenii Aleksandrovich Sevost'yanov and Ruslan Radikovich Salimov

Institute of Applied Mathematics and Mechanics of the NAS of Ukraine,

74, R. Luxemburg Str.,Donetsk 83114, Ukraine

E-Mail: brusin2006@rambler.ru, ruslan623@yandex.ru 